\title{
Comparison of motor competence in children aged 6-9 years across northern, central, and southern European regions
}

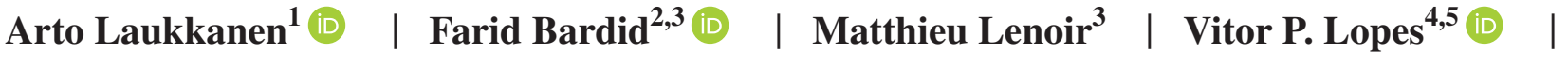 \\ Tommi Vasankari $^{6}$ | Pauliina Husu $^{6}$ (D) | Arja Sääkslahti ${ }^{1}$
}

${ }^{1}$ Faculty of Sport and Health

Sciences, University of Jyväskylä,

Jyväskylä, Finland

${ }^{2}$ School of Education, University of

Strathclyde, Glasgow, UK

${ }^{3}$ Department of Movement and Sports

Sciences, Ghent University, Ghent, Belgium

${ }^{4}$ Campus de Santa Apolónia, Instituto

Politécnico de Bragança, Bragança,

Portugal

${ }^{5}$ Research Centre of Sports, Health and Human Development (CIDESD), Portugal

${ }^{6}$ UKK Institute for Health Promotion

Research, Tampere, Finland

\section{Correspondence}

Arto Laukkanen, Faculty of Sport and Health Sciences, P.O. Box 35 (L366),

40014, University of Jyväskylä, Jyväskylä, Finland.

Email arto.i.laukkanen@jyu.fi

\section{Funding information}

Finnish Ministry of Education and Culture, Grant/Award Number: OKM/48/626/2014
The present study aimed at examining differences in motor competence (MC) in children aged 6-9 years old in northern, central, and southern European regions using the Körperkoordinationstest für Kinder (KTK). The secondary aim of the study was to examine interactions between region and children's age, sex, and weight status (determined as healthy weight or overweight/obese). Data were pooled from independent studies conducted in Finland (mean age $7.81 \pm 1.19$ years, $n=690$ ), Belgium (mean age $8.25 \pm 1.09$ years, $\mathrm{n}=1896$ ), and Portugal (mean age $8.31 \pm 1.02$ years, $\mathrm{n}=758$ ) between 2008 and 2016. Cross-cultural differences in MC and interaction effects were tested using ANOVAs and ANCOVAs. Age, sex, and BMI percentile were used as covariates. Geographical region significantly explained $19 \%$ of the variance in MC, while BMI (5\%), sex (3\%), and age $(0.3 \%)$ were significant covariates. The interaction effect of region and age (5\%), region and sex $(0.6 \%)$, and region and BMI $(0.2 \%)$ on MC was also significant. Cross-cultural differences in children's MC seem to increase substantially across 6 to 9 years, independent of the prevalence of overweight or obesity. Girls slightly underperformed in MC compared to boys in regions where the overall level of $\mathrm{MC}$ was lower. On the other hand, the association between body weight status and MC seems relatively consistent across the cultures. Future cross-cultural studies should further explore the influence of individual (eg, physical activity) and environmental (eg, physical activity and sport policy) factors on MC development.

\section{K E Y W O R D S}

childhood, cultural comparison, motor assessment, motor skills, movement skills

\section{1 | INTRODUCTION}

The prevalence of children classified as having poor gross motor competence (MC) has increased in recent decades. ${ }^{1-3}$ A study from a central European region showed that around $20 \%$ of children are "at-risk" of delays in motor development, ${ }^{3}$ while another study stated that the proportion of such children is more than $70 \%$ in the United States. ${ }^{4} \mathrm{MC}$ is used as an umbrella term reflecting different terminologies (eg, motor ability, fundamental movement/motor skills) that describe goal-directed human movement. ${ }^{5}$ An adequate level of $\mathrm{MC}$ enables one to take part in physical activities that are typical to one's age and developmental level. This is demonstrated by above-average physical activity levels ${ }^{6}$ achieving the daily recommended level of physical activity $(\mathrm{PA})^{4}$ and a higher likelihood of achieving a physically active lifestyle. ${ }^{7}$ These are globally topical issues, as the prevalence of children's inactivity is high ${ }^{8}$ and inactivity is associated with the 
increased prevalence of being overweight and obesity, as well as the accumulation of related health risks in children. ${ }^{9}$ Thus, there are social and public health needs for exploring reasons leading to population-level motor delay.

There is a dearth of international comparison studies on children's MC, even though cross-cultural studies might give insight into the cultural-environmental determinants of motor developmental differences. Existing inter-continental comparison studies have shown that children's MC differs significantly across the geographical regions. For instance, 6- to 8 -year-old Belgian children outperform their Australian peers in motor coordination, ${ }^{1} 4$ - to 5-year-old Belgian children outperform children in the United States in object control and locomotor skills, ${ }^{2}$ and 4- to 6-year-old Chinese children outperform their peers in the United States in manual dexterity and balance (although the children in the United States outperform their Chinese peers in throwing and catching). ${ }^{10}$ Differences have also been found in MC in 7- to 8-year-old children in Europe: northern European children (Norway) outperform southern European children (Greek and Italian) in fine and gross motor skills. ${ }^{11}$ It is speculated that crosscultural differences are due to differing enrollment rates in organized childcare ${ }^{1,2}$ and differences in policies governing physical education. ${ }^{2}$ The popularity of certain individual and group sports and games across nations may also influence the cross-cultural differences. For instance, Thomas et $\mathrm{al}^{12}$ showed that proficiency in a basic motor skill (throwing) is highly dependent on the prevailing sports culture.

However, reasons for cross-cultural differences in children's MC have remained speculative, in part because these studies used samples representing relatively narrow age ranges. From the perspective of motor development, it is essential to evaluate $\mathrm{MC}$ in a sample representing a large age range and to investigate whether interactions between $\mathrm{MC}$ and factors such as weight status change with age. For instance, being overweight has been found to predict a poor rate of MC development during primary school years. ${ }^{13}$ Secondly, previous studies have majorly compared childhood MC levels between countries from different continents, but it remains unclear if and to what extent motor competence levels differ among children from different parts of the same geographical region.

Therefore, to gain a better understanding of cross-cultural differences in MC among children in Europe, we pooled a considerable amount of data on northern, central, and southern European children aged 6-9 years $(\mathrm{N}=3460)$ living in Finland, Belgium, and Portugal. The first aim of the study was to investigate differences in MC between northern, central, and southern European cultures. The second and third aims were to examine interactions between geographical region and children's age and sex. We hypothesized that there are differences in children's MC across cultures ${ }^{11}$ and that these differences accumulate with age, as $\mathrm{MC}$ is shown to develop in childhood. ${ }^{14}$ Additionally, cultural influences may affect differences in MC between sexes. ${ }^{12}$ The fourth aim was to test whether body weight status, that is, being a healthy weight or overweight, influences MC differently across the geographical regions. There is substantial evidence showing an inverse association between body weight status and $\mathrm{MC},{ }^{5,15}$ and we hypothesized that this association might vary across the cultures and thus explain cross-cultural differences in MC. As a measure of MC, we used the Körperkoordinationstest fur Kinder (KTK), ${ }^{16,17}$ which is known to be a highly valid, reliable, and non-sport specific tool for assessing children's MC. ${ }^{18}$

\section{2 | MATERIALS AND METHODS}

\section{1 | Subjects}

This study pools data from four independent studies conducted between 2008 and 2016, all of which examined the levels of gross motor competence (MC) of children living in Finland, Belgium, and Portugal. The level of MC was assessed in each study using the KTK (Kiphard \& Schilling). ${ }^{16,17}$ These countries are culturally distinct and reflect different parts within Europe. Subjects from Finland (mean age $7.75 \pm 1.19$, range 4 years, $n=636$ ) were from two independent studies: the Skilled Kids study (years 2015-2016, mean age $6.64 \pm 0.36$ years, range 1.8 years, $n=278)$ and the Naantali Schools on the Move study (year 2013, mean age $8.60 \pm 0.85$ years, range 3.3 years, $n=412$ ). In the Skilled Kids study, children were enrolled at 37 childcare centers, which were geographically cluster-randomized around the whole country. In the Naantali study, children were enrolled at all 9 primary schools located in the city of Naantali and the principality of Masku in southwest Finland. Subjects were also drawn from 29 primary schools in the northern region of Belgium (ie, Flanders; year 2007; mean age $8.25 \pm 1.09$, range 4 years, $\mathrm{n}=1896$ ) and from eight primary schools in Portugal (year 2008; mean age $8.31 \pm 1.02$, range 3.9 years, $\mathrm{n}=758$ ) located in the central mainland region. Ethical approval was obtained from the Ethics Committees of the University of Jyväskylä and the Tampere Region (Finland), Ghent University Hospital (Belgium), and the University of Porto (Portugal). For each participant, written informed consent form was obtained from his or her parent or legal guardian.

\section{2 | Anthropometry}

Height and weight were measured in the childcare centers and schools to an accuracy of $0.1 \mathrm{~cm} / \mathrm{kg}$ by using professional-level scales (Finland: Charder HM 200P and Seca 877/Inbody 720; Belgium: Harpenden Portable Stadiometer, Holtain, UK and ANITA BC-420SM, Japan; Portugal: Seca 
217 and Seca 877). Body mass index (BMI) was calculated as weight $/$ height $^{2}\left(\mathrm{~kg} / \mathrm{m}^{2}\right)$. For each child, an age-adjusted $\mathrm{BMI}_{\text {percentile }}$ score was calculated using a Children's BMI Group Calculator-Metric Version, provided by the Centers for Disease Control and Prevention (CDC). ${ }^{19}$ According to the CDC norms, being overweight was defined as a BMI $\geq 85$ percentile and obesity as $\mathrm{BMI} \geq 95$ percentile.

\section{3 | Motor competence}

MC was tested by teams of trained observers using the KTK. ${ }^{16,17}$ This is a product-oriented assessment tool appropriate for use with 5- to 14-year-old children who are typically developing as well as those with brain damage, behavioral problems, or learning difficulties. The KTK assesses gross motor coordination and body control, mainly the dynamic balance instead of single motor skills. The KTK has been used widely for research purposes in Europe over the last four decades, ${ }^{18}$ and it has been used in criterion validity studies of other assessment tools, such as Movement Assessment Battery for Children (M-ABC). ${ }^{20,21}$

As part of the KTK test battery, the children performed the following four tasks:

1. Walking backwards (WB) on balance beams (length $3 \mathrm{~m}$, height $5 \mathrm{~cm}$ ) with different widths of $6.0,4.5$, and $3.0 \mathrm{~cm}$, starting with the widest one. The maximum test score possible was 72 steps, based on 3 trials per each beam and a maximum of 8 successful steps for each trial.

2. Hopping for height $(\mathrm{HH})$, one foot at a time, over an increasing pile of soft mattresses (width $60 \mathrm{~cm}$; depth $20 \mathrm{~cm}$; height $5 \mathrm{~cm}$ each). The first, second, or third trial of each height was awarded by three, two, or one point(s), respectively. The maximum test score was 39 points (ground level +12 mattresses) for each leg, resulting in a maximum of 78 points with both legs.

3. Jumping sideways (JS) from side to side over a thin wooden lath $(60 \times 4 \times 2 \mathrm{~cm})$ on a jumping base $(100 \times 60 \mathrm{~cm})$. Two trials of 15 seconds were performed, and the total of successful jumps was calculated.

4. Moving sideways (MS). The children had two identical wooden plates (size $25 \times 25 \mathrm{~cm}$, height $5.7 \mathrm{~cm}$ ), and after stepping to one, they had to transfer another one sideways for the next transition. The total of transitions was summed over two 20-second trials. Transitions were performed in the same direction on both trials.

The psychometric properties of the KTK have been welldocumented; content and construct validity have been established ${ }^{16,17}$ Additionally, the KTK has been shown to moderately correlate with other widely used assessment tools such as the M-ABC $(r=0.62-0.65)^{20}$ and the Bruininks-Oseretsky Test of
Motor Proficiency (BOT-2) Short Form $(r=0.61-0.64) .{ }^{21,22}$ The KTK protocol has shown moderate-to-high reliability based on test-retest correlation $(r=0.60-0.99)^{23-26}$ and high reliability based on inter-rater correlation $(r=0.90-0.99) .{ }^{24} \mathrm{In}$ addition, Cronbach's alphas for the four items showed a high internal consistency (0.95). ${ }^{24}$ The raw test scores of the KTK test items were transformed into sex- and age-standardized values and into a measure indicating overall gross $\mathrm{MC}$ according to the renewed norms. ${ }^{27} \mathrm{MC}$ scores were classified as follows: "not possible" (values under 56), "impaired" (values 56-70), “poor” (values 71-85), "typical” (values 86-115), "good” (values 116-130), and "very good" (values 131-145).

\section{4 | Statistical analyses}

Statistical analyses were performed using the statistical software package IBM SPSS version 24.0. Children with one or more missing MC items (Belgium, $\mathrm{n}=209$ ) and children without birth date information (Portugal, $\mathrm{n}=127$ ) were excluded from the analyses. Means and standard deviations of sample characteristics (age, height, weight, BMI, BMI ${ }_{\text {percentile, being }}$ overweight $\%$, obesity $\%$, and $\mathrm{MC}$ ) were obtained and the differences between sexes tested using independent sample $t$ tests (age, height, weight, and $\mathrm{BMI}_{\text {percentile }}$ ) and chi-square $\left(\chi^{2}\right)$ tests $\left(\mathrm{BMI}_{\text {healthy/overweight or obese }}\right)$. Differences between the regions in terms of age and anthropometric descriptives were tested using one-way analysis of variance (ANOVA) and one-way analysis of covariance (ANCOVA) when adjusted for age. Internal consistency of the norm-based KTK test item values was examined by calculating Cronbach's alphas and item-total correlations separately for the three regions. A 2 (sex) $\times 4$ (age-group, age rounded down to the nearest integer) ANOVA was used to examine the age and sex differences in the standardized KTK values and MC within the regions. MC scores were plotted with age separately for healthy-weight, overweight, and obese children in each region. Linear regression lines were calculated and drawn to illustrate the linear relationships. Furthermore, Pearson correlations were calculated between the MC scores and age in each of these subgroups.

A $\chi^{2}$ test was used to compare the distributions of children from the three regions across the KTK performance categories (impaired, poor, typical, good, very good). ANCOVAs were performed to examine regional differences in MC. Firstly, the effect of geographical region (Finland, Belgium, and Portugal) on $\mathrm{MC}$ was examined with age and $\mathrm{BMI}_{\text {percentile }}$ as covariates using a one-way ANCOVA (Model 1). Twoway ANCOVAs were performed where interaction effects were examined: region $\times$ age-group (Model 2), region $\times$ sex (Model 3), and lastly, region $\times$ BMI status group (Model 4). The covariates used were $\mathrm{BMI}_{\text {percentile }}$ and sex (Model 2), age and $\mathrm{BMI}_{\text {percentile }}($ Model 3), and age and sex (Model 4). Covariates were chosen on the basis of previous evidence 
indicating their effect on $\mathrm{MC}$ in children. ${ }^{3} \mathrm{BMI}_{\text {percentile was }}$ chosen as a covariate instead of weight or height because it had the strongest correlation with MC when adjusted for age. Significant interaction and main effects were further investigated with the Bonferroni post-hoc tests or pairwise comparisons. The level of significance was set as $P<.05$ in all analyses.

\section{RESULTS}

\section{1 | Sample characteristics}

Age and anthropometric statistics showed that boys overall were significantly taller than girls in Finland $(t=2.09$, $P<.05)$ and Belgium $(t=2.79, P<.01)$ (Table 1$)$. There were significant differences between the regions in age $(F=56.12, P<.001, \mathrm{FI}<\mathrm{BE} / \mathrm{PT})$, height $(F=13.89$, $P<.001$, FI $<$ BE $<$ PT $)$, weight $(F=39.08, P<.001$, FI $<$ BE $<$ PT), BMI $(F=38.59, P<.001, \mathrm{FI} / \mathrm{BE}<\mathrm{PT})$, $\mathrm{BMI}_{\text {percentile }}(F=33.07, P<.001, \mathrm{BE}<\mathrm{FI} / \mathrm{PT})$, prevalence of being overweight (Pearson $\chi^{2}=71.11, d f=2, P<.001$, $\mathrm{BE}<\mathrm{FI}<\mathrm{PT}$ ), and obesity prevalence (Pearson $\chi^{2}=33.72$, $d f=2, P<.001, \mathrm{BE}<\mathrm{FI}<\mathrm{PT})$. Differences in anthropometric variables between regions were mainly due to differences in the sample mean ages. After adjustment for age, an ANCOVA revealed that the region significantly explained no more than $1 \%-3 \%$ of the variation in anthropometry.

Cronbach's alphas of the KTK test items were 0.828 , 0.804, and 0.777 in samples from Finland, Belgium, and Portugal, respectively. The corrected item-total correlations for the norm-based values of test items of the walking backwards, hopping for height, jumping sideways, and moving sideways, respectively, were as follows: .571, .710, .695, .655 (Finland); .549, .656, .687, .588 (Belgium); .648, .578, .680, .616 (Portugal). Performance in the KTK norm-based values was significantly higher in older age-groups in Finland, remained the same in Belgium, and was significantly lower in Portugal (Table 2, Figure 1). Age-group affected 15.9\%, $0.3 \%$, and $16.0 \%$ of the variation in MC in Finland, Belgium, and Portugal, respectively. Overall, boys outperformed girls in $\mathrm{MC}$ in all three regions. However, sex explained $11.7 \%$ of the variation in $\mathrm{MC}$ in the Portuguese group but only $2.0 \%$ and $0.9 \%$ in the Belgian and Finnish groups, respectively. For the hopping for height task, the age $\times$ sex interaction effect was significant in the Belgian and Portuguese samples and borderline significant in the Finnish sample. For the moving sideways task, the age $\times$ sex interaction effect was significant in the Belgian group.

$\mathrm{BMI}_{\text {percentile }}$ was negatively associated with $\mathrm{MC}$ in all three regions: Finland $(r=-0.131)$, Belgium $(r=-0.228)$, and Portugal ( $r=-0.316)$ (Figure 2). Healthy-weight children had positive associations between age and MC in Finland and Belgium but not in Portugal. Interestingly, all the subgroups of healthy-weight, overweight, and obese children showed an increasing trend of MC according to age in Finland, whereas only healthy-weight children showed an increasing trend of MC with age in Belgium, and all the subgroups showed a decreasing trend of $\mathrm{MC}$ according to age in Portugal.

Differences in motor competence between geographical regions.

A $\chi^{2}$ test showed a significant difference in the distribution of children in the three regions across the KTK performance categories $\left(\chi^{2}=459.984 ; P<.001\right)$ (Figure 3$)$. The majority of children in each of the regions were rated as typically developing. However, those in Portugal who were not rated as being typically developing were rated mostly as having poor MC (29.3\%) or impaired MC (10.3\%); only $0.9 \%$ scored above the typical range. In comparison, a remarkable proportion of Finnish and Belgian children were categorized as being above the typical range (33.8\% and $18.4 \%$ ) and minority of the children as being below the typical range $(8.7 \%$ and $10.8 \%$ ), respectively.

The one-way ANCOVA showed a significant main effect of region on MC, explaining approximately $19 \%$ of the variance (Table 3, Model 1). The results also showed significant main effects for the covariates age, $\mathrm{BMI}_{\text {percentile, and sex, }}$ which explained $0.03 \%, 4.8 \%$, and $2.9 \%$ of the variance in MC, respectively. Pairwise comparisons between regions revealed that Portuguese children scored $21.46 \%$ and $16.63 \%$ lower in MC compared to Finnish (adjusted mean difference $-18.93, P<.001$ ) and Belgian children (adjusted mean difference $-13.60, P<.001$ ), respectively. Finnish children scored $5.11 \%$ higher in $\mathrm{MC}$ compared to Belgian children (adjusted mean difference 5.76, $P<.001$ ).

Model 2 indicated that there was a statistically significant region $\times$ age-group interaction effect for MC, explaining around $5 \%$ of the variance overall. Pairwise comparisons showed that the region $\times$ age-group interaction explained $10.6 \%, 2.5 \%$, and $2.4 \%$ of the variability in MC between Finland and Portugal, between Finland and Belgium, and between Belgium and Portugal, respectively (all $P<.001$ ). Finnish children outperformed Belgian and Portuguese children at ages 7, 8, and 9, while Belgian children outperformed Portuguese children at ages 6 to 9 and Finnish children at age 6.

The region $\times$ sex interaction was found to be statistically significant although very weak, explaining only approximately $1 \%$ of the variation in MC. Pairwise comparisons showed that region $\times$ sex interaction was significant between Finland and Portugal and between Belgium and Portugal (both $P<.001$ ), although these explained only $1 \%$ of the variation in $\mathrm{MC}$ in both comparisons. Adjusted means of MC were $2.36 \%, 3.69 \%$, and $9.03 \%$ lower in girls compared to boys in Finland, Belgium, and Portugal, respectively.

Region $\times \mathrm{BMI}_{\text {healthy/overweight or obese }}$ showed a statistically significant interaction effect on $\mathrm{MC}$; however, this only 
TA B L E 1 Descriptive statistics (means \pm standard deviations) of age and anthropometric measurements stratified by region, age-group, and sex

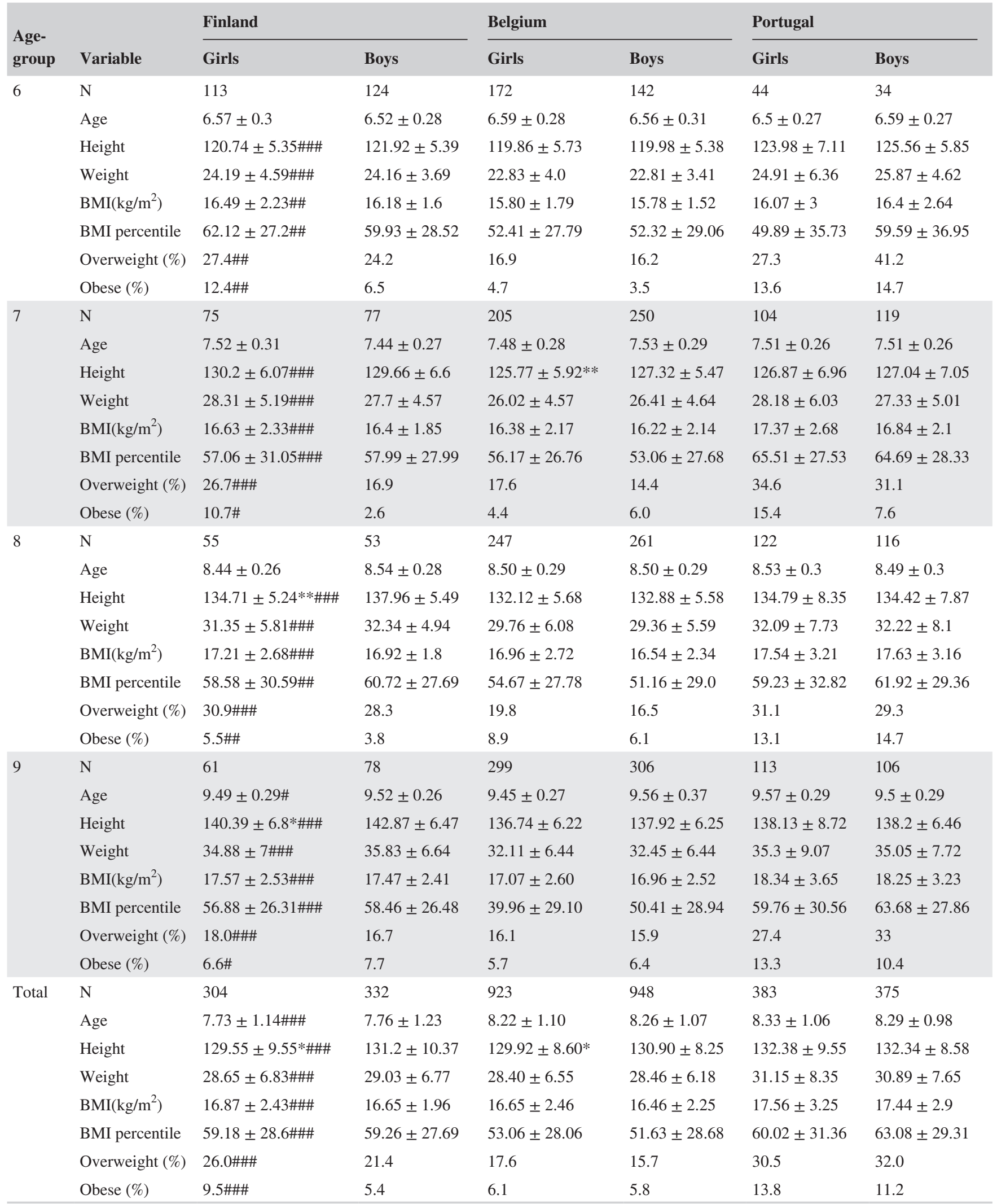

Note: *Significant difference between sexes at the level $P<.05$; **Significant difference between sexes at the level $P<.01$; ${ }^{*}$ Significant difference between regions at

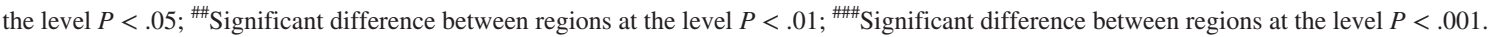


TA B L E 2 Standardized values (mean \pm standard deviation) of the four items of the KTK and the total test battery of the Finnish, Belgian, and Portuguese boys and girls for all the age-groups

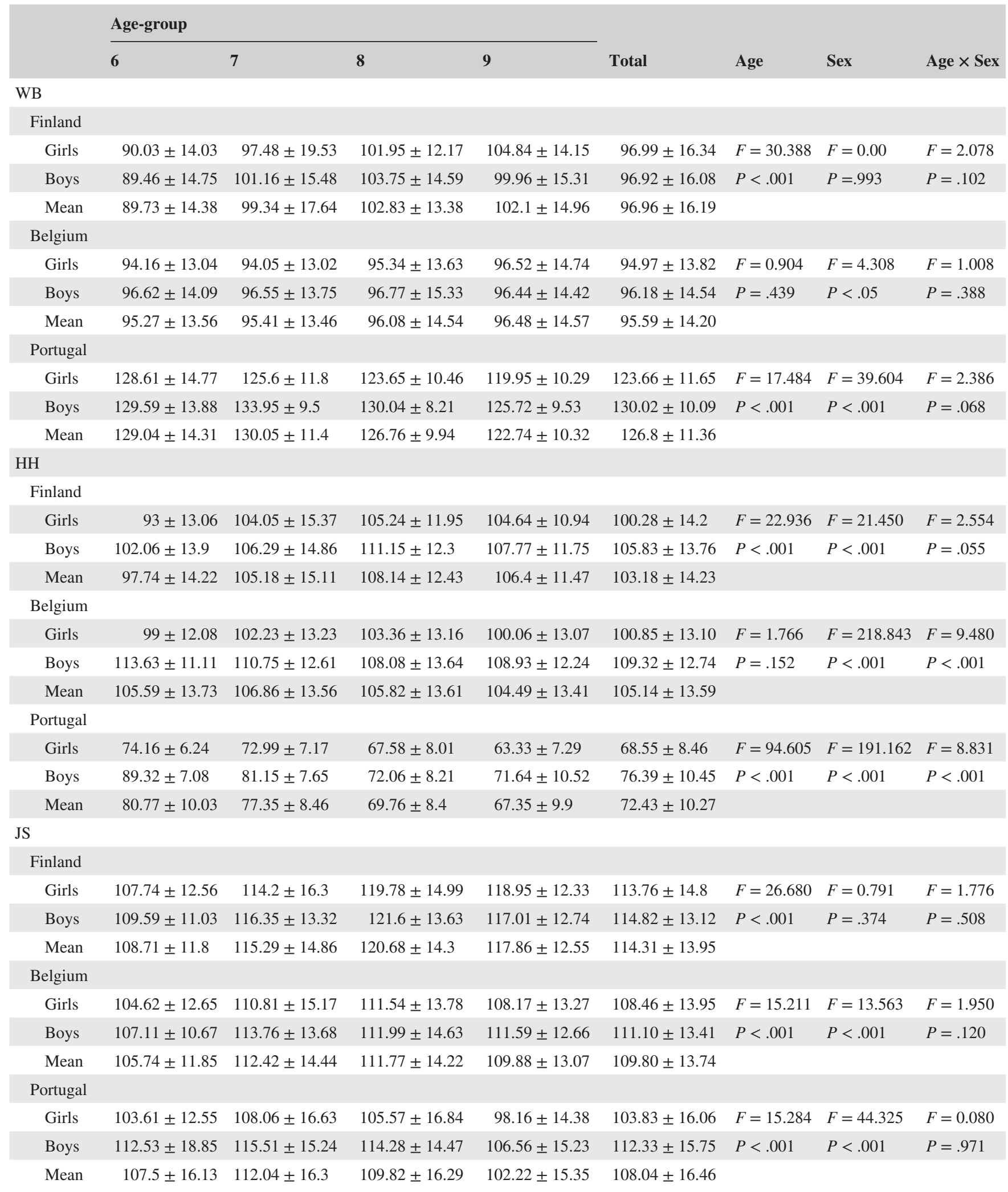


T A B L E 2 (Continued)

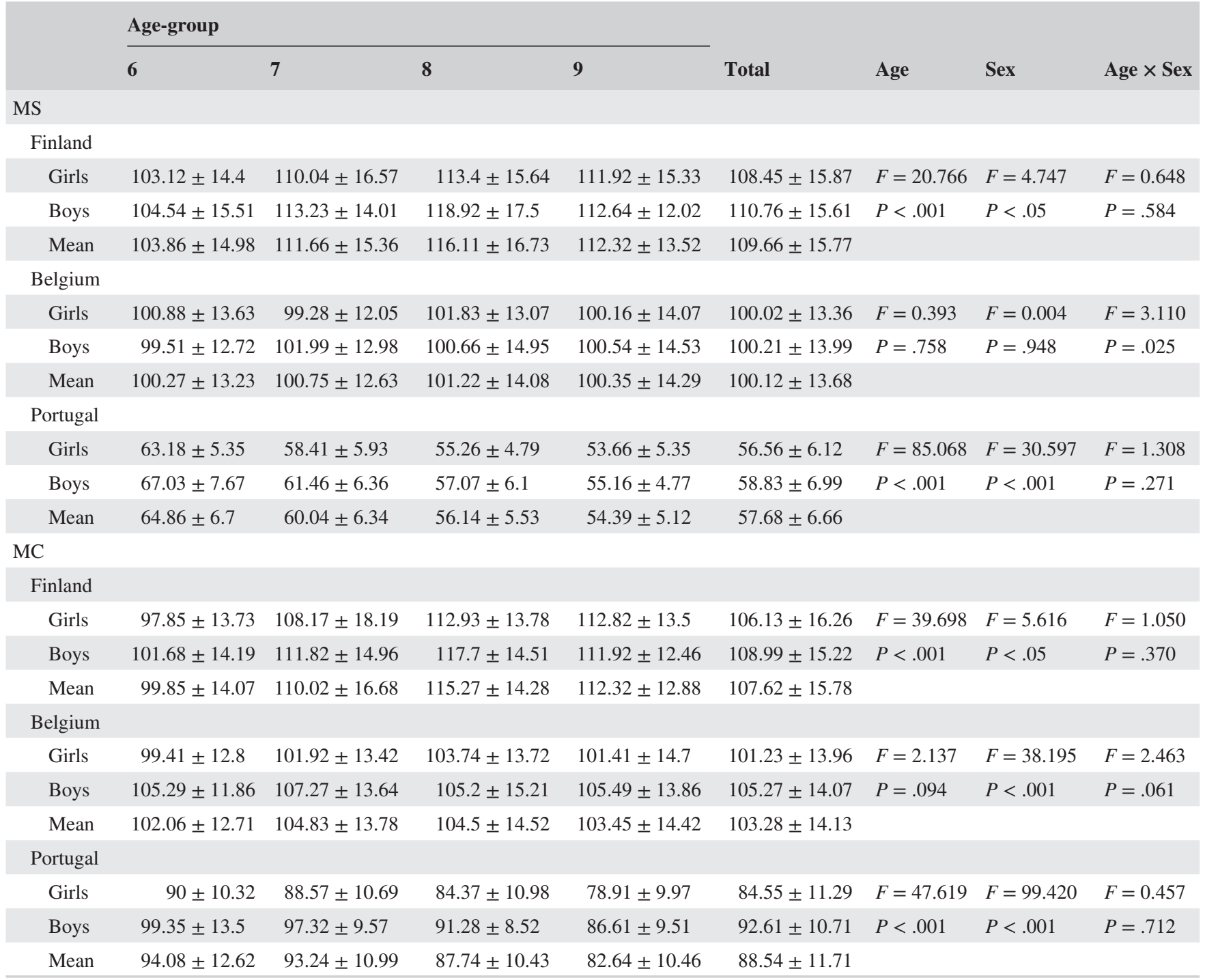

Note: Abbreviations: HH, hopping for height; JS, jumping sideways; KTK, KörperkoordinationsTest für Kinder; MC, motor competence; MS, moving sideways; WB, walking backwards.

explained $0.2 \%$ of the variance (Table 3, Model 4). Pairwise comparisons showed that the region $\times \mathrm{BMI}_{\text {healthy/overweight or }}$ obese interaction was statistically significant between Finland and Belgium $(P<.05)$ and between Finland and Portugal $(P<.05)$, although they only explained $0.2 \%$ and $0.4 \%$ of the variation in $\mathrm{MC}$, respectively. Adjusted means of $\mathrm{MC}$ were $5.92 \%, 10.2 \%$, and $12.07 \%$ lower for overweight or obese children compared to the healthy-weight peers in Finland, Belgium, and Portugal, respectively.

\section{4 | DISCUSSION}

The results of this study reveal considerable differences in 6- to 9-year-old children's MC across northern, central, and southern European regions, suggesting that cross-cultural differences are due essentially to differences in the developmental rate of MC throughout childhood. Additionally, the study suggests that these differences may weakly relate to girls', and on the other hand, overweight children's greater underperformance in MC compared to boys' and healthyweight children's in regions where the overall level of MC is lower. Overall, the results suggest that motor development in childhood is influenced by individual and environmental factors, although only a small part of that variation is explained in the present study.

The cross-cultural differences found in the present study confirm the findings of a previous cross-cultural comparison, which used the same MC assessment. The study conducted by Bardid and colleagues ${ }^{1}$ indicated significantly greater MC in Belgian children compared to Australian children, while the children's BMI and age were identified as significant 


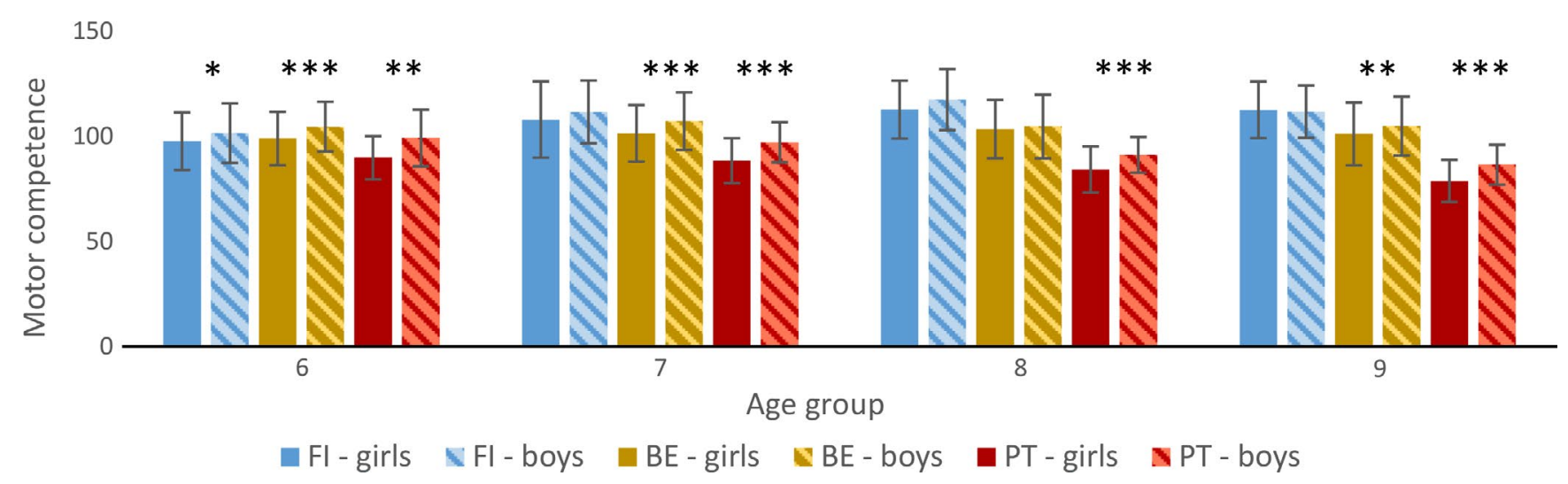

F I G URE 1 The comparison of motor competence between the sexes in Finland, Belgium, and Portugal. * Significant sex difference at the level of $P<.05$; ** Significant sex difference at the level of $P<.01$; *** Significant sex difference at the level of $P<.001$. FI $=$ Finland; $\mathrm{BE}=$ Belgium; $\mathrm{PT}=$ Portugal

covariates of MC. Bardid et $\mathrm{al}^{1}$ also found that cross-cultural differences in $\mathrm{MC}$ accumulated significantly between the ages of 6 and 8 years, yet their explained variability was smaller than that of the present study ( $2 \%$ vs $5 \%$ ). This difference is probably due to greater cross-cultural differences in $\mathrm{MC}$ and the broader age range examined in the present study. In contrast to previous cross-cultural comparisons, ${ }^{1,2,10}$ the present study showed that boys' and girls' differing MCs significantly, although rather weakly, explain cross-cultural differences in MC. Specifically, the sex difference in MC was greater in cultures where the overall level of MC is lower. A unique finding of the present study showing statistically significant interaction between geographical region and BMI category should be interpreted practically trivial because of a very low effect size.

From Newell's constraints-based perspective, there are constraints relating to individual, environmental, or task factors which inhibit motor development. ${ }^{28}$ These factors
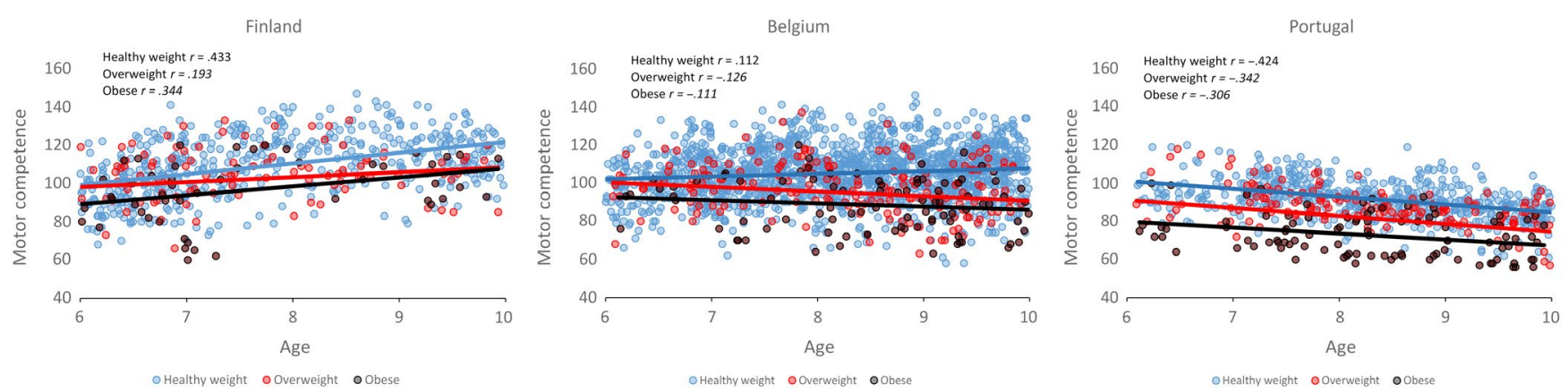

F I G U RE 2 Scatter plot of the association between age and motor competence for healthy-weight, overweight, and obese Finnish, Belgian, and Portuguese children. Pearson correlation coefficients are presented for each body weight category

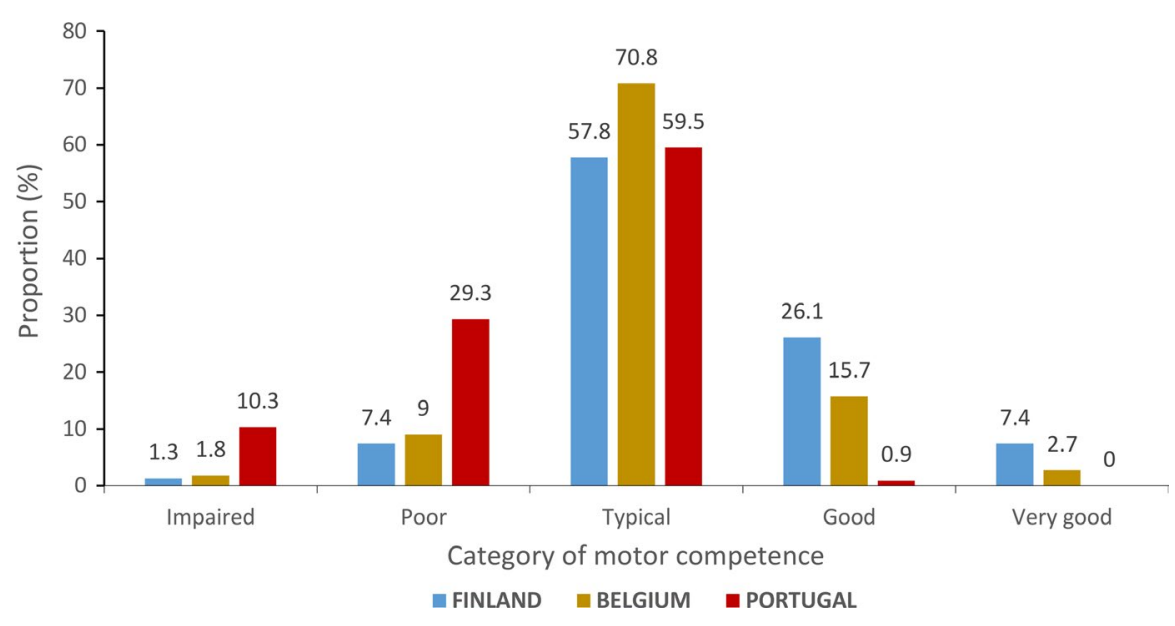

F IG URE 3 Proportion of children across KTK performance ratings for the three geographical regions 
TABLE 3 Main and interaction effects on motor competence according to geographical region, age, body mass index, and sex

\begin{tabular}{|c|c|c|c|c|c|c|}
\hline & $\begin{array}{l}\text { Type III } \\
\text { sum of } \\
\text { squares }\end{array}$ & $d f$ & Mean square & $\boldsymbol{F}$ & Sig & $\eta_{p}^{2}$ \\
\hline \multicolumn{7}{|l|}{ Model 1 (ANCOVA) } \\
\hline Region & 142635,633 & 2 & 71317,816 & 392,119 & 0.000 & 0.194 \\
\hline Age (covariate) & 1686,581 & 1 & 1686,581 & 9,273 & 0.002 & 0.003 \\
\hline $\mathrm{BMI}_{\text {percentile }}$ (covariate) & 30189,825 & 1 & 30189,825 & 165,990 & 0.000 & 0.048 \\
\hline Sex (covariate) & 17811,403 & 1 & 17811,403 & 97,931 & 0.000 & 0.029 \\
\hline \multicolumn{7}{|c|}{ Model 2 (2-way ANCOVA) } \\
\hline Region & 122441,798 & 2 & 61220,899 & 360,060 & 0.000 & 0.181 \\
\hline $\mathrm{Age}_{6-9-\text { years }}$ & 7226,833 & 3 & 2408,944 & 14,168 & 0.000 & 0.013 \\
\hline $\mathrm{BMI}_{\text {percentile }}$ (covariate) & 30137,419 & 1 & 30137,419 & 177,248 & 0.000 & 0.052 \\
\hline Sex (covariate) & 17111,363 & 1 & 17111,363 & 100,638 & 0.000 & 0.030 \\
\hline Region $\times$ Age $_{6-9 \text {-years }}$ & 29386,325 & 6 & 4897,721 & 28,805 & 0.000 & 0.050 \\
\hline \multicolumn{7}{|c|}{ Model 3 (2-way ANCOVA) } \\
\hline Region & 142696,093 & 2 & 71348,047 & 394,484 & 0.000 & 0.195 \\
\hline Age (covariate) & 1757,145 & 1 & 1757,145 & 9,715 & 0.002 & 0.003 \\
\hline $\mathrm{BMI}_{\text {percentile }}$ (covariate) & 30721,824 & 1 & 30721,824 & 169,861 & 0.000 & 0.050 \\
\hline Sex & 16112,949 & 1 & 16112,949 & 89,089 & 0.000 & 0.027 \\
\hline Region $\times$ Sex & 3665,137 & 2 & 1832,569 & 10,132 & 0.000 & 0.006 \\
\hline \multicolumn{7}{|c|}{ Model 4 (2-way ANCOVA) } \\
\hline Region & 115957,686 & 2 & 57978,843 & 326,444 & 0.000 & 0.167 \\
\hline Age (covariate) & 1923,222 & 1 & 1923,222 & 10,829 & 0.001 & 0.003 \\
\hline $\begin{array}{l}\mathrm{BMI}_{\text {healthy/overweight or }} \\
\text { obese }\end{array}$ & 35084,598 & 1 & 35084,598 & 197,540 & 0.000 & 0.057 \\
\hline Sex (covariate) & 16932,491 & 1 & 16932,491 & 95,337 & 0.000 & 0.028 \\
\hline $\begin{array}{l}\text { Region } \times \mathrm{BMI}_{\text {healthy } /} \\
\text { overweight or obese }\end{array}$ & 1225,683 & 2 & 612,842 & 3,451 & 0.032 & 0.002 \\
\hline
\end{tabular}

Abbreviations: ANCOVA, analysis of covariance; BMI, body mass index; Region, geographical region. influence motor development through their mutual interaction, although some of the factors may be more important than others at a given time. Body weight status is known to act as an individual-level constraint to motor development, ${ }^{13,15}$ and the negative association found between $\mathrm{BMI}_{\text {percentile }}$ and $\mathrm{MC}$ in all the three regions of the present study confirms this assumption. We hypothesized that the association between BMI and MC may vary across the cultures, which may explain cross-cultural differences in MC. This hypothesis was partly supported as the correlation between $\mathrm{BMI}_{\text {percentile }}$ and $\mathrm{MC}$ varied across the cultures, but the geographical region $\times \mathrm{BMI}$ interaction effect on $\mathrm{MC}$ was found very weak in magnitude. In practice, overweight or obese children are more likely having a low absolute level of MC in cultures where the overall level of MC is lower but the difference between healthy-weight and overweight or obese children's MC seems to differ only marginally across the cultures. The findings overall support an assumption that the association between being overweight or obese and having a poor MC is relatively consistent across the cultures. Other individual-level factors which might explain $\mathrm{MC}$ differences, in addition to being overweight, could be PA and fitness levels. Previously, children's cardiorespiratory fitness has been shown to differ across countries, similar to the differences in $\mathrm{MC}$ found in the present study. ${ }^{29}$ Poor fitness levels may act as an individual constraint for motor development in childhood, for instance, through lower perceived sports competence ${ }^{30}$ and therefore less participation in PA typical of a subject's age and developmental level.

Based on the Global Matrix 2.0 on PA, ${ }^{31}$ Finland and Belgium are considered to outperform Portugal in some major indicators, namely active play, active transportation, community and built environment, and government strategies and investments for PA. Active play, especially if performed outdoors, is associated with greater moderate-to-vigorous PA and lower sedentary levels,${ }^{32}$ thus contributing to motor development. ${ }^{6}$ The total amount of active play also influences the amount of very short-term and high-intensive PA impacts which are known to contribute to the differences in MC. ${ }^{33}$ Secondly, active transportation in children goes hand-in-hand 
with children's independent mobility, an issue in which Finland is the world's leading country and Portugal at the end of the list. ${ }^{34}$ Furthermore, children's independent mobility and active transportation are both largely dependent on community and built environment, an issue which separates Finland's and Portugal's PA indicators the most. ${ }^{31}$ The results of the present study showed that sex differences in MC are larger when the overall level of MC in the culture is lower, an issue which may reflect cultural differences in attitudes toward the importance of PA for boys and girls. It is well known that boys tend to be more physically active compared to girls ${ }^{35}$ and that boys naturally participate in a wider variety of PA games compared to girls. ${ }^{36}$ Gender equality has strong historical roots in northern European countries, ${ }^{37}$ and the more equal levels of $\mathrm{MC}$ in girls and boys especially in Finland may be a reflection of national emphasis on gender equality. Interestingly, this hypothesis is supported by the fact that, internationally, poor gender equality is associated with lower grades in PA indicators. ${ }^{31}$ Finland's greater effort with regard to government strategies and investments compared to Portugal and Belgium may further explain cross-cultural differences in children's MC, although this needs further investigation. In contrast, Portugal is considered to outperform both Finland and Belgium in organized sport participation, ${ }^{31}$ and this can be seen, for instance, in systematic and organized school sport competitions at local and national levels in Portugal. ${ }^{38}$ Additionally, time allocated to physical education in primary schools is officially higher in Portugal compared to Finland and Belgium. Nonetheless, it should be noted that generalist classroom teachers teach physical education lessons in primary schools in years 1-4 in Portugal. Physical education lessons in primary schools are generally taught by specialist physical education teachers in Belgium and partly by generalist teachers and specialists physical education teachers in Finland, a fact which may enhance quality of physical education and development of children's MC. ${ }^{38,39}$ Finally, remarkable climate differences across Europe may affect cross-cultural differences in KTK performance. Seasonal variation in temperature is associated with children's PA and motor development, ${ }^{14}$ and it may be that Finnish children in particular benefit from the long winter season with snow (eg, cross country skiing) and ice (eg, ice skating) when it comes to the KTK test, which strongly emphasizes performance in tasks relating to gross motor coordination and dynamic balance. Overall, multiple individual (eg, PA, physical fitness) and environmental (eg, organized physical activity and sport, physical education) factors may explain the cross-cultural differences in children's MC and should be explored in future studies.

\section{1 $\quad$ Strengths and limitations}

The strengths of this study are the substantial sample size gathered for each geographical region evaluated and the use of a non-sport-specific, reliable, and valid MC assessment. Although in each of the substudies great care was taken to collect data from a representative sample, it cannot be excluded that representativeness was equal and optimal in all subsamples. In addition, cross-sectional study design represents a substantial limitation for the interpretation of the study results. Observing children of varying ages at one time point creates a risk of misinterpretation, because we cannot really know how children will develop over time. Furthermore, the data collections are up to 9 years apart. Culture-specific phenomena may have taken place, such as physical activity campaigns, which may have affected levels of MC among some groups evaluated in this study. On the other hand, KTK does not assess object control skills nor fine motor skills. Nonetheless, KTK can be considered as a good measure of MC as it has shown to correlate moderately with test batteries including gross and fine motor skills as well as manipulative skills. ${ }^{20-22}$ Another limitation is the low number of background variables used in the study. There are several variables which may explain cross-cultural differences in MC, such as parental support ${ }^{14}$ and peer support for $\mathrm{PA},{ }^{40}$ as well as, children's physical activity level and participation in organized PA and sports. ${ }^{6}$ On the other hand, this is one of the first studies that brings cross-cultural differences of MC to the attention and thus opens the gateway for further research. Lastly, the data have a multi-level structure (subjects in classes, classes in schools, schools in countries), but statistical modeling was done by using single-level structure (subjects), a fact that may blur associations between the factors.

\section{5 | PERSPECTIVE}

This present study suggests that the developmental rate of MC substantially differs among children aged 6-9 years between northern, central, and southern European regions. Alarmingly, around $40 \%$ of children from southern Europe might have poor or impaired motor competence, compared to around $10 \%$ of children from northern and central Europe. The results also indicate that these differences are independent of the remarkable cross-cultural differences in body weight status. Other key findings suggest that girls are slightly more likely to underperform in $\mathrm{MC}$ compared to boys when the overall level of MC in the region is lower. However, the inverse association between body weight status and MC seems relatively consistent across the cultures. Overall, only a small part of the cross-cultural variation in children's MC was successfully explained in the present study. The substantial cross-cultural differences in children's MC may be due to several individual and environmental factors, such as active play in childhood, gender equality, and 
government strategies for and investments in PA. These cultural factors should be further explored in future research.

\section{ORCID}

Arto Laukkanen (iD https://orcid.org/0000-0002-9722-0258

Farid Bardid (D) https://orcid.org/0000-0001-8591-0596

Vitor P. Lopes (D) https://orcid.org/0000-0003-1599-2180

Pauliina Husu (D) https://orcid.org/0000-0003-1502-7428

Arja Sääkslahti (D) https://orcid.org/0000-0003-4354-0990

\section{REFERENCES}

1. Bardid F, Rudd JR, Lenoir M, Polman R, Barnett LM. Cross-cultural comparison of motor competence in children from Australia and Belgium. Front Psychol. 2015;6:694.

2. Brian A, Bardid F, Deconinck F, Lenoir M, Goodway J. Actual and perceived motor competence levels of Belgian and US preschool children. J Mot Learn Dev. 2017;1-29.

3. Vandorpe B, Vandendriessche J, Lefevre J, et al. The Körperkoordinationstest für Kinder: reference values and suitability for 6-12-year-old children in Flanders. Scand J Med Sci Sports. 2011;21(3):378-388.

4. De Meester A, Stodden D, Goodway J, et al. Identifying a motor proficiency barrier for meeting physical activity guidelines in children. J Sci Med Sport. 2018;21(1):58-62.

5. Robinson LE, Stodden DF, Barnett LM, et al. Motor competence and its effect on positive developmental trajectories of health. Sport Med. 2015;45(9):1273-1284.

6. Holfelder B, Schott N. Relationship of fundamental movement skills and physical activity in children and adolescents: a systematic review. Psychol Sport Exerc. 2014;15(4):382-391.

7. Lloyd M, Saunders TJ, Bremer E, Tremblay MS. Long-term importance of fundamental motor skills: a 20-year follow-up study. Adapt Phys Act Q. 2014;31:67-78.

8. Hallal PC, Andersen LB, Bull FC, Guthold R, Haskell W, Ekelund U. Global physical activity levels: surveillance progress, pitfalls, and prospects. Lancet. 2012;380(9838):247-257.

9. Ng M, Fleming T, Robinson M, et al. Global, regional and national prevalence of overweight and obesity in children and adults 1980 2013: a systematic analysis. Lancet. 2014;384(9945):766-781.

10. Chow SMK, Henderson SE, Barnett AL. the movement assessment battery for children: a comparison of 4-year-old to 6-year- old children from Hong Kong and the United States. Am J Occup Ther. 2001;55(1):55-61.

11. Haga M, Tortella P, Asonitou K, et al. Cross-cultural aspects: exploring motor competence among 7- to 8-year-old children from Greece, Italy, and Norway. SAGE Open. 2018;8(2):1-9

12. Thomas JR, Alderson JA, Thomas KT, Campbell AC, Elliott BC. Developmental gender differences for overhand throwing in Aboriginal Australian children. Res $Q$ Exerc Sport. 2010;81(4):432-441.

13. Rodrigues LP, Stodden DF, Lopes VP. Developmental pathways of change in fitness and motor competence are related to overweight and obesity status at the end of primary school. J Sci Med Sport. 2016;19(1):87-92.
14. Laukkanen A, Pesola AJ, Heikkinen R, Sääkslahti AK, Finni T. Family-based cluster randomized controlled trial enhancing physical activity and motor competence in 4-7-year-old children. PLoS ONE. 2015;10(10):1-17.

15. D'Hondt E, Deforche B, Gentier I, et al. A longitudinal study of gross motor coordination and weight status in children. Obesity. 2014;22(6):1505-1511.

16. Kiphard EJ, Schilling F. KörperkoordinationsTest Für Kinder. Weinheim, Germany: Beltz-Verlag; 1974.

17. Kiphard EJ, Schilling F. KörperkoordinationTest Für Kinder (2nd edn). Göttingen, Germany: Belzt test; 2007.

18. Iivonen S, Sääkslahti AK, Laukkanen A. A review of studies using the Körperkoordinationstest für Kinder (KTK). Eur J Adapt Phys Act. 2015;8(2):18-36.

19. Centers for Disease Control and Prevention. Children's BMI tool for schools. . Accessed: 2017-04-27. (Archived by WebCite ${ }^{\circledR}$ at https://www.cdc.gov/healthyweight/assessing/bmi/childrens_bmi/ tool_for_schools.htmlhttp://www.webcitation.org/6q2D51GY1). Published 2015.

20. Henderson SE, Sugden DA. Movement Assessment Battery for Children. London, UK: Psychological Corporation; 1992.

21. Smits-Engelsman BCM, Henderson SE, Michels CGJ. The assessment of children with developmental coordination disorders in the Netherlands: the relationship between the movement assessment battery for children and the Körperkoordinations Test für Kinder. Hum Mov Sci. 1998;17(4-5):699-709.

22. Fransen J, D'Hondt E, Bourgois J, Vaeyens R, Philippaerts RM, Lenoir M. Motor competence assessment in children: convergent and discriminant validity between the BOT-2 short form and KTK testing batteries. Res Dev Disabil. 2014;35(6):1375-1383.

23. Bardid F, Huyben F, Deconinck FJA, De Martelaer K, Seghers J, Lenoir M. Convergent and divergent validity between the KTK and MOT 4-6 motor tests in early childhood. Adapt Phys Act $Q$. 2016;33(1):33-48.

24. Camacho-Araya T, Woodburn SS, Boschinini C. Reliability of the prueba de coordinacion corporal para ninos (body coordination test for children). Percept Mot Skills. 1990;70(3):832-834.

25. Freitas DL, Lausen B, Maia JA, et al. Skeletal maturation, fundamental motor skills and motor coordination in children 7-10 years. J Sports Sci. 2015;33(9):924-934.

26. Lopes VP, Rodrigues LP, Maia JAR, Malina RM. Motor coordination as predictor of physical activity in childhood. Scand J Med Sci Sports. 2011;21(5):663-669.

27. Kiphard EJ, Schilling F. KTK Körperkoordinationstest Für Kinder (Body Coordination Test). 3., überar. (Schilling F, ed.). Göttingen: Hogrefe Verlag Gmbh; 2017.

28. Newell KM. Constraints on the Development of Coordination. In: Wade M, Whiting H, eds. Motor Development in Children: Aspects of Coordination and Control. Amsterdam, the Netherlands: Marting Nijhoff; 1986:341-361.

29. Lang JJ, Tremblay MS, Léger L, Olds T, Tomkinson GR. International variability in $20 \mathrm{~m}$ shuttle run performance in children and youth: who are the fittest from a 50-country comparison? A systematic literature review with pooling of aggregate results. $\mathrm{Br}$ J Sports Med. 2018;52(4):276.

30. Smith JJ, Eather N, Morgan PJ, Plotnikoff RC, Faigenbaum AD, Lubans DR. The health benefits of muscular fitness for children and adolescents: a systematic review and meta-analysis. Sport Med. 2014;44(9):1209-1223. 
31. Tremblay MS, Gonzalez SA, Katzmarzyk PT, Onywera VO, Reilly JJ, Tomkinson G. Introduction to the global matrix 2.0: report card grades on the physical activity of children and youth comparing 38 countries. J Phys Act Heal. 2016;13(11 suppl 2):S85-S86.

32. Larouche R, Garriguet D, Tremblay MS. Outdoor time, physical activity and sedentary time among young children: the 2012-2013 Canadian health measures survey. Can J Public Heal. 2016;107(6):e500-e506.

33. Laukkanen A, Pesola A, Havu M, Sääkslahti A, Finni T. Relationship between habitual physical activity and gross motor skills is multifaceted in 5- to 8-year-old children. Scand J Med Sci Sports. 2014;24(2):e102-110.

34. Shaw B, Bicket M, Elliott B, Fagan-Watson B, Mocca E, Hillman M. Children's Independent Mobility: An International Comparison and Recommendations for Action. London, UK: Policy Studies Institute; 2015.

35. Bauman AE, Reis RS, Sallis JF, Wells JC, Loos RJF, Martin BW. Correlates of physical activity: Why are some people physically active and others not? Lancet. 2012;380(9838):258-271.

36. Storli R, Sandseter EBH. Preschool teachers' perceptions of children's rough-and-tumble play (R\&T) in indoor and outdoor environments. Early Child Dev Care. 2015:1-15.
37. OECD. Is the Last Mile the Longest? Economic Gains from Gender Equality in Nordic Countries. Paris, France: OECD Publishing; 2018.

38. Hardman K. Current situation and prospects for physical education in the European Union. Brussels: European Parliament; 2007. http://www.europarl.europa.eu/RegData/etudes/etudes/ join/2007/369032/IPOL-CULT_ET(2007)369032_EN.pdf

39. Katsarova I. Physical Education in EU Schools. Brussels: European Parliament; 2016. http://www.europarl.europa.eu/RegData/etudes/ BRIE/2016/593559/EPRS_BRI(2016)593559_EN.pdf

40. Lopes VP, Gabbard C, Rodrigues LP. Effects of psychosocial variables in the similarity and interdependence of physical activity levels among adolescent best friend dyads. J Sports Sci. 2016;34(9):821-828.

How to cite this article: Laukkanen A, Bardid F, Lenoir M, et al. Comparison of motor competence in children aged 6-9 years across northern, central, and southern European regions. Scand J Med Sci Sports. 2019;00:1-12. https://doi.org/10.1111/sms.13578 